\title{
Simultaneous integrated boost intensity-modulated radiotherapy versus 3-dimensional conformal radiotherapy in preoperative concurrent chemoradiotherapy for locally advanced rectal cancer
}

\author{
Bong Kyung Bae, MD', Min Kyu Kang, MD', Jae-Chul Kim, MD, PhD', \\ Mi Young Kim, MD², Gyu-Seog Choi, MD, PhD³, Jong Gwang Kim, MD, PhD, \\ Byung Woog Kang, MD, PhD ${ }^{4}$, Hye Jin Kim, MD, PhD ${ }^{3}$, Soo Yeun Park, MD, PhD ${ }^{3}$ \\ ${ }^{1}$ Department of Radiation Oncology, Kyungpook National University School of Medicine, Daegu; ${ }^{2}$ Department of Radiation \\ Oncology, Kyungpook National University Chilgok Hospital, Daegu; ${ }^{3}$ Colorectal Cancer Center, Kyungpook National University \\ Chilgok Hospital, Kyungpook National University School of Medicine, Daegu; ${ }^{4}$ Department of Oncology/Hematology, Kyungpook \\ National University Chilgok Hospital, Kyungpook National University School of Medicine, Daegu, Korea
}

Purpose: To evaluate the feasibility of simultaneous integrated boost intensity-modulated radiotherapy (SIB-IMRT) for preoperative concurrent chemoradiotherapy (PCRT) in locally advanced rectal cancer (LARC), by comparing with 3-dimensional conformal radiotherapy (3D-CRT).

Materials and Methods: Patients who were treated with PCRT for LARC from 2015 January to 2016 December were retrospectively enrolled. Total doses of $45 \mathrm{~Gy}$ to $50.4 \mathrm{~Gy}$ with 3D-CRT or SIB-IMRT were administered concomitantly with 5-fluorouracil plus leucovorin or capecitabine. Surgery was performed 8 weeks after PCRT. Between PCRT and surgery, one cycle of additional chemotherapy was administered. Pathologic tumor responses were compared between SIB-IMRT and 3D-CRT groups. Acute gastrointestinal, genitourinary, hematologic, and skin toxicities were compared between the two groups based on the RTOG toxicity criteria.

Results: SIB-IMRT was used in 53 patients, and 3D-CRT in 41 patients. After PCRT, no significant differences were noted in tumor responses, pathologic complete response ( $9 \%$ vs. 7\%; $p=1.000)$, pathologic tumor regression Grade 3 or higher $(85 \%$ vs. 71\%; $p=$ $0.096)$, and R0 resection ( $87 \%$ vs. 85\%; $p=0.843$ ). Grade 2 genitourinary toxicities were significantly lesser in the SIB-IMRT group ( $8 \%$ vs. $24 \% ; p=0.023$ ), but gastrointestinal toxicities were not different across the two groups.

Conclusion: SIB-IMRT showed lower GU toxicity and similar tumor responses when compared with 3D-CRT in PCRT for LARC.

Keywords: Rectal neoplasms, Intensity-modulated radiotherapy, Conformal radiotherapy, Chemoradiotherapy, Neoadjuvant therapy

Received 06 July 2017, Revised 24 July 2017, Accepted 31 July 2017.

Correspondence: Min Kyu Kang, MD, Department of Radiation Oncology, Kyungpook National University Chilgok Hospital, 807 Hoguk-ro, Buk-gu, Daegu 41404, Korea. Tel: +82-53-200-2653, Fax: +82-53-200-2029, E-mail: mkkang@knu.ac.kr

(c) This is an Open Access article distributed under the terms of the Creative Commons Attribution Non-Commercial License (http://creativecommons.org/ licenses/by-nc/4.0/) which permits unrestricted non-commercial use, distribution, and reproduction in any medium, provided the original work is properly cited.

www.e-roj.org 


\section{Introduction}

Preoperative chemoradiotherapy (PCRT) followed by surgery is the preferred standard of treatment for locally advanced rectal cancer (LARC) [1]. Radiotherapy (RT) field for LARC is recommended to encompass the primary tumor, entire mesorectum, presacral space, and regional lymphatics. Traditionally, 2D techniques with 3- to 4-fields were used for coverage of the region of interest. After the introduction of 3-dimensional conformal radiotherapy (3D-CRT), dose coverage of target areas and sparing of organs at risks (OARs) has been improved [2]. However, it is difficult to avoid OARs even with 3D-CRT because of the horseshoe-shaped target volume, surrounding the small bowel and the bladder, in rectal cancer [3]. To further decrease irradiated volumes and doses delivered to OARs, more advanced RT techniques such as intensity-modulated radiotherapy (IMRT) are needed.

IMRT has been widely used for various cancer sites, such as head and neck cancer and prostate cancer. Even though the dosimetric advantages of IMRT over conformal RT have been reported in rectal cancer [4-6], the comparisons of clinical outcomes between two techniques have not been reported much. In addition to the ability to spare the doses of OARs, IMRT can deliver different doses to different target volumes in a single fraction. This simultaneous integrated boost IMRT (SIB-IMRT) can increase the fraction size of boost volume with a lower dose to the elective volume, providing clinical and dosimetric advantages $[7,8]$. There are several phase II clinical trials of PCRT for LARC using SIB-IMRT, reporting good oncologic outcomes with acceptable toxicities [9-12]. Based on these clinical data, we commenced SIB-IMRT for PCRT in LARC.

To evaluate the safety and efficacy of SIB-IMRT in LARC, early treatment outcomes and acute toxicities were compared between the 3D-CRT and SIB-IMRT groups.

\section{Materials and Methods}

The Institutional Review Boards of Kyungpook National University Chilgok Hospital (No. 2017-06-023) and Kyungpook National University Hospital (No. 2017-06-012-001) approved this retrospective study and waived the requirement for informed patient consent.

\section{Patients}

From 2015 January to 2016 December, 116 patients were treated with the intention of PCRT for LARC. The patients who met the following inclusion criteria were included in this study: patients with pathologically proven adenocarcinoma, patients with locally advanced disease (CT3 or higher T stage, or positive lymph node), patients treated with 3D-CRT or SIB-IMRT, and patients who underwent surgery in our institution. Clinical staging of patients was based on initial chest and abdomen computed tomography (CT), pelvic magnetic resonance imaging (MRI), and positron emission tomography-computed tomography (PET-CT). Patients who underwent surgery outside our institution, refused surgery, or did not complete planned PCRT were excluded. Finally, 94 patients met the criteria and were evaluated for the analysis.

\section{Radiotherapy}

Planning CT with a 2- to 5-mm slice thickness was performed in the prone position for 3D-CRT, and in the supine position for SIB-IMRT. Patients were instructed to hold urination for 1 hour before simulation and each treatment session. The general principles of target contouring were based on pre-existing guidelines, which were identical in 3D-CRT and SIB-IMRT $[3,13]$. The gross tumor volume (GTV) was defined as a tumor visible on CT and MRI. Fusion of diagnostic pelvic MRI and planning CT scans was optionally carried out for contouring assistance. Clinical target volume 1 (CTV1) started from the L5S1 junction, encompassing the internal iliac lymphatic region, the obturator lymphatic region, the presacral area, and the mesorectum down to the pelvic floor. When visceral organs anterior to the rectum were involved by tumor infiltration, the external iliac lymphatic regions were also included in CTV1. CTV2 for boost was defined as the entire mesorectum area at the level of GTV plus $1 \mathrm{~cm}$, or the GTV plus $3 \mathrm{~mm}$, based on the practitioner's preferences. To consider the inter-fractional variation of target volume according to the stool, bowel gas, and bladder filling, additional anterior margin of 5-10 $\mathrm{mm}$ was added to CTV. Finally, 5-6 mm margins from CTV1 and CTV2 were added for planning target volumes (PTV1 and PTV2).

RT doses and techniques had changed during the study period. Total dose of 3D-CRT was escalated from 45 Gy in 25 fractions to $50.4 \mathrm{~Gy}$ in 28 fractions from January 2016. After April 2016, we commenced to apply SIB-IMRT of $50 \mathrm{~Gy}$ in 25 fractions to PCRT. Forty-one patients were treated with 3D-CRT using 3-field or 4-field box techniques. After receiving $45 \mathrm{~Gy}$ in 25 fractions to PTV1, 21 patients received boost of $5.4 \mathrm{~Gy}$ in 3 fractions to PTV2. Fifty-three patients were treated with SIB-IMRT; patients received 45 Gy in 25 fractions to PTV1, and $50 \mathrm{~Gy}$ in 25 fractions to PTV2. SIB-IMRT was generated with a dynamic multileaf collimator technique using 7-9 fields, or a volumetric arc technique using 2 arcs. For the SIB-IMRT 
planning, the dose distribution in the normal tissue outside the PTV1/2 was set to fall off rapidly. The maximum dose of the small bowel and bladder outside the PTV1/2 was kept to be lower than the prescribed doses. And, the volume of the small bowel and bladder receiving 30-40 Gy was further reduced.

During the treatment course of 3D-CRT and SIB-IMRT, the position of each patient was verified using on-board kilovoltage imaging device or electronic portal imaging device once a week.

\section{Chemotherapy and surgery}

Either 5-fluorouracil (5-FU) plus leucovorin or capecitabine was used for concurrent chemotherapy during RT. 5-FU (425 $\mathrm{mg} / \mathrm{m}^{2}$, days $\left.1-4\right)$ plus leucovorin $\left(20 \mathrm{mg} / \mathrm{m}^{2}\right.$, days $\left.1-4\right)$ was intravenously administered during the first and fifth weeks of RT. Capecitabine $\left(825 \mathrm{mg} / \mathrm{m}^{2}\right)$ was administered twice daily on the day of RT for 25 days. One additional infusion of the previously described chemotherapy regimen of 5 -FU plus leucovorin was administered 3 weeks after the end of CRT.

Surgery with total mesorectal excision was performed median 8 weeks after PCRT. Varied surgical techniques were employed based on patient's status and surgeon's preferences, including lower anterior resection, abdominoperineal resection, and intersphincteric resection.

\section{Evaluation and statistical analysis}

Pathologic responses based on surgical pathology were compared between 3D-CRT and SIB-IMRT groups for the evaluation of tumor responses, including primary tumor downstaging, nodal downstaging, pathologic tumor regression grade (TRG) based on Dworak grading system [14], and pathologic complete response (pCR) defined as ypTONO. Clinical and pathologic tumor staging was based on the American Joint Committee on Cancer 7th edition [15]. Dworak grading system is as follows: Grade 0 , no regression; Grade 1, dominant tumor mass with obvious fibrosis and/or vasculopathy; Grade 2 , dominantly fibrotic changes with few tumor cells or groups (easy to identify); Grade 3, very few (difficult to identify microscopically) tumor cells in fibrotic tissue with or without mucous substance; and Grade 4, no tumor cells, only fibrotic mass (total regression or response).

During RT, all patients attended weekly interview with radiation oncologists. For the evaluation of acute toxicities related to PCRT and surgery, patient charts were reviewed for gastrointestinal (GI), genitourinary (GU), hematologic, and skin toxicities, and for early anastomotic leakage. GI events included abdominal pain, constipation, diarrhea, increased fecal frequency, painful defecation, and tenesmus. GU events included increase in urinary frequency, dysuria, and urinary urgency. Hematologic toxicities were graded based on laboratory results of white blood cell (WBC) and platelet counts, and hemoglobin levels. Skin toxicity was graded based on visual inspection and subjective complains of the patient. Early leakage was defined as leakage that required additional intervention within 1 month after surgery. Adverse events were graded based on the Radiation Therapy Oncology Group toxicity criteria.

To compare dose-volume parameters of the small bowel and the bladder between 3D-CRT and SIB-IMRT groups, individual loops of the small bowel and the entire bladder were delineated at the level of PTV. Volume receiving $15 \mathrm{~Gy}$ or more $\left(V_{15}\right)$, volume receiving 45 Gy or more $\left(V_{45}\right)$, maximum dose $\left(D_{\text {max }}\right)$, and mean dose $\left(D_{\text {mean }}\right)$ of the small bowel and the bladder were evaluated.

To compare the tumor responses and toxicities between the 3D-CRT and SIB-IMRT groups, Pearson chi-square test, Fisher exact test, and t-test were used depending on the variables. IBM SPSS ver. 23 (IBM Corp., Armonk, NY, USA) was used for analysis, and a p-value of less than 0.05 was considered statistically significant.

\section{Results}

\section{Characteristics}

Table 1 shows the baseline patient, tumor, and treatment characteristics. The median age of all patients was 64 years (range, 24 to 85 years). Eighty-one patients (86\%) were diagnosed with stage III disease. All 53 patients in the SIBIMRT group received $50 \mathrm{~Gy}$, while 20 patients in the 3D-CRT group (49\%) received 45 Gy. The proportion of patients with capecitabine for PCRT was higher in the SIB-IMRT group than in the 3D-CRT group (70\% vs. 49\%; $p=0.038$ ). The median time from the end of PCRT to surgery was 58 days (range, 38 to 105 days). Age, gender, performance status, stage, mesorectal fascia involvement, distance from the anal verge, time to surgery, and type of surgery were not significantly different between the SIB-IMRT and 3D-CRT groups (all p-values $>0.05$ ).

\section{Tumor responses}

Table 2 shows the pathologic stages after PCRT. pCR was observed in 8 patients (9\%). Eleven patients (12\%) had stage disease, 32 patients (34\%) had stage II, and 43 patients (46\%) had stage III. The distributions of stages were not different 
Table 1. Baseline patient, tumor, and treatment characteristics

\begin{tabular}{|c|c|c|c|c|}
\hline & All & 3D-CRT & SIB-IMRT & $p$-value \\
\hline Age (yr) & $64(24-85)$ & $61(32-85)$ & $65(24-83)$ & 0.229 \\
\hline Gender & & & & 0.281 \\
\hline Male & $73(78)$ & $34(83)$ & $39(74)$ & \\
\hline Female & $21(22)$ & $7(17)$ & $14(26)$ & \\
\hline ECOG performance status & & & & 0.109 \\
\hline 0 & $41(44)$ & $13(32)$ & $28(53)$ & \\
\hline 1 & $50(53)$ & $26(63)$ & $24(45)$ & \\
\hline 2 & $3(3)$ & $2(5)$ & $1(2)$ & \\
\hline Tumor grade & & & & 0.046 \\
\hline Well differentiated & $23(24)$ & $14(34)$ & $9(17)$ & \\
\hline Moderately differentiated & $46(49)$ & $16(39)$ & $30(57)$ & \\
\hline Poorly differentiated & $4(4)$ & $0(0)$ & $4(8)$ & \\
\hline Unknown & $21(22)$ & $11(27)$ & $10(19)$ & \\
\hline Clinical stage & & & & 0.163 \\
\hline$\|$ & $13(14)$ & $5(12)$ & $8(15)$ & \\
\hline III & $81(86)$ & $36(88)$ & $45(85)$ & \\
\hline cT & & & & 0.300 \\
\hline cT2 & $3(3)$ & $0(0)$ & $3(6)$ & \\
\hline cT3 & $67(71)$ & $30(73)$ & $37(70)$ & \\
\hline cT4 & $24(26)$ & $11(27)$ & $13(25)$ & \\
\hline $\mathrm{cN}$ & & & & 0.686 \\
\hline Negative & $13(14)$ & $5(12)$ & $8(15)$ & \\
\hline Positive & $81(86)$ & $36(88)$ & $45(85)$ & \\
\hline Mesorectal fascia & & & & 0.544 \\
\hline Involved & $61(65)$ & $28(68)$ & $33(62)$ & \\
\hline Not involved & $33(35)$ & $13(32)$ & $20(38)$ & \\
\hline Distance from the anal verge $(\mathrm{cm})$ & $5.6 \pm 2.8$ & $5.7 \pm 2.6$ & $5.6 \pm 2.9$ & 0.933 \\
\hline$\leq 5$ & $43(46)$ & $20(49)$ & $23(43)$ & 0.603 \\
\hline$>5$ & $51(54)$ & $21(51)$ & $30(57)$ & \\
\hline Total dose (Gy) & & & & $<0.001$ \\
\hline 50.4 or 50 & $74(79)$ & $21(51)$ & $53(100)$ & \\
\hline 45 & $20(21)$ & $20(49)$ & $0(0)$ & \\
\hline Chemotherapy & & & & 0.038 \\
\hline 5-fluorouracil + leucovorin & $37(39)$ & $21(51)$ & $16(30)$ & \\
\hline Capecitabine & $57(61)$ & $20(49)$ & $37(70)$ & \\
\hline Time to surgery (day) & $58(38-105)$ & $57(44-72)$ & $59(38-105)$ & 0.141 \\
\hline Type of surgery & & & & 0.237 \\
\hline Low anterior resection & $54(57)$ & $24(59)$ & $30(57)$ & \\
\hline Abdominoperineal resection & $5(5)$ & $0(0)$ & $5(9)$ & \\
\hline Intersphincteric resection & $35(37)$ & $17(41)$ & $18(34)$ & \\
\hline
\end{tabular}

Values are presented as median (range) or number (\%) or mean \pm standard deviation.

3D-CRT, three-dimensional conformal radiotherapy; SIB-IMRT, simultaneous integrated boost intensity-modulated radiotherapy; ECOG, Eastern Cooperative Oncology Group.

between the SIB-IMRT and 3D-CRT groups.

Primary tumor downstaging was observed in 39 patients (41\%). Of the 81 patients with clinically positive lymph node, 40 patients (49\%) showed conversion to a negative lymph node status. Tumor response of TRG 3-4 was seen in 74 patients (79\%). When comparing tumor responses between the two groups, no significant differences were observed (Table 3).

\section{Toxicities}

There was no Grade 3 or higher toxic events reported on GI, $\mathrm{GU}$, and skin toxicity profiling. Three patients experienced Grade 3 hematologic toxic events: 2 related with WBC counts and 1 related with the hemoglobin level. The incidence of Grade $2 \mathrm{GU}$ toxicity was significantly lower in the SIB-IMRT group, while that of other toxicity grades were not different 
Table 2. Pathologic stages after preoperative chemoradiotherapy followed by surgery

\begin{tabular}{|c|c|c|c|c|}
\hline & All & 3D-CRT & SIB-IMRT & $p$-value \\
\hline ypStage & & & & 0.986 \\
\hline 0 (pCR) & $8(9)$ & $3(7)$ & $5(9)$ & \\
\hline I & $11(12)$ & $5(12)$ & $6(11)$ & \\
\hline$\|$ & $32(34)$ & $14(34)$ & $18(34)$ & \\
\hline III & $43(46)$ & $19(46)$ & $24(45)$ & \\
\hline урт & & & & 0.981 \\
\hline TO & $8(9)$ & $3(7)$ & $5(9)$ & \\
\hline T1-2 & $14(15)$ & $6(15)$ & $8(15)$ & \\
\hline T3-4 & $72(77)$ & $32(78)$ & $40(75)$ & \\
\hline ypN & & & & 0.919 \\
\hline Negative & $51(54)$ & $22(54)$ & $29(55)$ & \\
\hline Positive & $43(46)$ & $19(46)$ & $24(45)$ & \\
\hline
\end{tabular}

Values are presented as number (\%).

3D-CRT, three-dimensional conformal radiotherapy; SIB-IMRT, simultaneous integrated boost intensity-modulated radiotherapy; pCR, pathologic complete response.

Table 3. Tumor responses to preoperative chemoradiotherapy

\begin{tabular}{|c|c|c|c|c|}
\hline & All & 3D-CRT & SIB-IMRT & p-value \\
\hline Primary tumor downstaging & & & & 0.670 \\
\hline Yes & $39(41)$ & $16(39)$ & $23(43)$ & \\
\hline No & $55(59)$ & $25(61)$ & $30(57)$ & \\
\hline Nodal downstaging & & & & 0.960 \\
\hline Yes & $40(49)$ & $18(50)$ & $22(49)$ & \\
\hline No & $41(51$ & $18(50)$ & $23(51)$ & \\
\hline Completeness of resection & & & & 0.843 \\
\hline Ro & $81(86)$ & $35(85)$ & $46(87)$ & \\
\hline $\mathrm{R} 1$ & $13(14)$ & $6(15)$ & $7(13)$ & \\
\hline Pathologic tumor regression grade & & & & 0.096 \\
\hline $1-2$ & $20(21)$ & $12(29)$ & $8(15)$ & \\
\hline $3-4$ & $74(79)$ & $29(71)$ & $45(85)$ & \\
\hline
\end{tabular}

Values are presented as number (\%).

3D-CRT, three-dimensional conformal radiotherapy; SIB-IMRT, simultaneous integrated boost intensity-modulated radiotherapy.

between the groups. After surgery, 8 patients (9\%) experienced early leakage requiring additional intervention with no difference in patient distribution between the two groups. Details of acute toxicities are summarized in Table 4.

On comparing dose-volume parameters, $V_{45}$ of the small bowel and the bladder were significantly lower in the SIB-IMRT group. However, there were no differences in other parameters. Details are shown in Table 5.

\section{Discussion and Conclusion}

This study compared early clinical outcomes between two RT techniques using similar strategies on tumor dose and target volumes. Tumor responses were not different between SIB-
IMRT and 3D-CRT. The incidence of GU toxicities of Grade 2 was significantly lower in the SIB-IMRT group; however, GI toxicities were not different between the two groups on the contrary to our expectation.

The rate of pCR after PCRT has been reported to be 10.4\%27\% [16-19]. In the current study, the pCR rate was 9\% with no difference between the 3D-CRT and SIB-IMRT groups. The previous study from our institution, with the CRT regimens similar to the current study, showed a pCR rate of 22.7\% [20]. Even though the reason for this difference is unclear, the differences between the cohorts might explain this; in the earlier investigation, patients were treated during 2010-2013 vs. 2015-2016 in the current one, and also the proportion of patients with cT4 doubled from 13\% to $26 \%$ during this 
Table 4. Comparison of acute toxicities between 3D-CRT and IMRT groups

\begin{tabular}{|c|c|c|c|c|c|}
\hline & Grade & All & 3D-CRT & SIB-IMRT & $p$-value \\
\hline \multirow[t]{2}{*}{ Gastrointestinal } & $0-1$ & 83 (88) & 37 (90) & $46(87)$ & 0.751 \\
\hline & 2 & $11(12)$ & 4 (10) & 7 (13) & \\
\hline \multirow[t]{2}{*}{ Genitourinary } & $0-1$ & $80(85)$ & 31 (76) & $49(92)$ & 0.023 \\
\hline & 2 & $14(15)$ & $10(24)$ & 4 (8) & \\
\hline \multirow[t]{2}{*}{ Hematologic } & $0-1$ & 73 (78) & $30(73)$ & $43(81)$ & 0.358 \\
\hline & $2-3$ & $21(22)$ & $11(27)$ & $10(19)$ & \\
\hline \multirow[t]{2}{*}{ Skin } & $0-1$ & $70(74)$ & $27(66)$ & $43(81)$ & 0.092 \\
\hline & 2 & $24(26)$ & $14(34)$ & $10(19)$ & \\
\hline \multirow[t]{2}{*}{ Early leakage } & Absent & 86 (91) & 38 (93) & $48(91)$ & 1.000 \\
\hline & Present & $8(9)$ & $3(7)$ & $5(9)$ & \\
\hline
\end{tabular}

Values are presented as number (\%).

3D-CRT, three-dimensional conformal radiotherapy; SIB-IMRT, simultaneous integrated boost intensity-modulated radiotherapy.

Table 5. Comparison of dose-volume parameters of the small bowel and the bladder between the 3D-CRT and SIB-IMRT groups

\begin{tabular}{|c|c|c|c|}
\hline & $3 \mathrm{D}-\mathrm{CRT}(\mathrm{n}=41)$ & SIB-IMRT $(n=53)$ & $p$-value \\
\hline \multicolumn{4}{|l|}{ Small bowel } \\
\hline$V_{15}\left(\mathrm{~cm}^{3}\right)$ & $172.0 \pm 160.7$ & $223.0 \pm 150.4$ & 0.117 \\
\hline$V_{45}\left(\mathrm{~cm}^{3}\right)$ & $38.1 \pm 44.5$ & $8.5 \pm 10.3$ & $<0.001$ \\
\hline $\mathrm{D}_{\text {mean }}(\mathrm{Gy})$ & $23.6 \pm 7.3$ & $24.7 \pm 8.2$ & 0.478 \\
\hline$D_{\max }(G y)$ & $45.5 \pm 9.3$ & $43.1 \pm 13.4$ & 0.326 \\
\hline \multicolumn{4}{|l|}{ Bladder } \\
\hline$V_{15}\left(\mathrm{~cm}^{3}\right)$ & $116.6 \pm 102.4$ & $140.7 \pm 98.8$ & 0.251 \\
\hline$V_{45}\left(\mathrm{~cm}^{3}\right)$ & $32.4 \pm 36.5$ & $15.6 \pm 17.3$ & 0.004 \\
\hline $\mathrm{D}_{\text {mean }}(\mathrm{Gy})$ & $34.2 \pm 6.1$ & $33.6 \pm 5.1$ & 0.562 \\
\hline$D_{\max }(G y)$ & $48.1 \pm 2.5$ & $48.9 \pm 6.4$ & 0.468 \\
\hline
\end{tabular}

Values are presented as mean \pm standard deviation.

3D-CRT, three-dimensional conformal radiotherapy; SIB-IMRT, simultaneous integrated boost intensity-modulated radiotherapy.

period. In patients who did not achieved pCR, TRG could be an excellent alternative factor for the assessment of patients' prognosis $[19,21,22]$. Our rate of TRG 3-4 (79\%) was relatively higher than those in other studies (38.6\%-63\%) [19,21,22], even though direct comparisons among different studies are difficult. When we compared the rates of TRG 3-4 between SIB-IMRT and 3D-CRT groups, no significant differences were observed (85\% vs. 71\%; $p=0.096$ ).

As IMRT has been widely used in many cancer sites to reduce normal tissue toxicities, IMRT has been attempted for rectal cancer. Some authors have reported that the incidence of Grade 2 or higher GI toxicity was significantly reduced with IMRT vs. 3D-CRT (30\%-32\% vs. 61\%-62\%) [23,24]. Parekh et al. [24] noted that Grade 2 or higher diarrhea reduced with IMRT vs. 3D-CRT (10\% vs. 43\%; $p=0.014$ ) in PCRT for rectal cancer. However, a prospective multi-institutional single arm study of PCRT for rectal cancer concluded that IMRT could not reduce $\mathrm{GI}$ toxicity, with a result of Grade 2 or higher $\mathrm{GI}$ toxicity in $51.5 \%$ of the patients [25]. In the current study, there was no difference in Grade $2 \mathrm{GI}$ toxicity between the SIB-IMRT and 3D-CRT groups (13\% vs. 10\%). There are several possible reasons for this. Even though $V_{45}$ of the small bowel was significantly smaller in the SIB-IMRT group, $V_{15}$ of the small bowel was not different between the two groups, which was reported to be a strong factor for acute small bowel toxicity [26]. In addition, it was not possible to capture or discriminate all PCRT-related toxicities because of the retrospective nature of this study. The difference in concurrent chemotherapeutic agents could have affected the results (Table 1). As all the symptoms existing before treatment were not recorded, we could not use the Common Terminology Criteria for Adverse Events criteria and this restricts the direct comparison of our results with other studies.

Acute Grade $2 \mathrm{GU}$ toxicities were significantly lower in the SIB-IMRT group compared to the 3D-CRT group (4\% vs. 24\%; $p=0.023$ ). Reduced doses of the bladder and urethra would 
be considered for this difference. Dose volume effects on bladder toxicity in pelvic RT have been more focused on highdose radiation and late toxicities [27], so the presence of acute toxicities related to lower dose is relatively unclear. Although the clinical significance is unknown, SIB-IMRT showed advantages in bladder sparing in $\mathrm{V}_{45}$ of the bladder. When reviewing the dose distribution near the proximal urethra, SIBIMRT seemed to give a lower dose to the area where 3D-CRT would be supposed to give a dose similar to the prescribed dose because of the geometric relationship between both internal iliac areas and the proximal urethra; a connecting line of the most anterior portions of internal iliac nodal regions is anterior to that of the rectum and traverses the area near the proximal urethra.

Although pCR is known to be an important factor of good prognosis after PCRT in LARC and some studies have reported dose-response relationship in PCRT [28-31], the optimal RT dose for PCRT is still unclear. Appelt et al. [30] revealed a significant dose-response relationship in the range of $50.4 \mathrm{~Gy}$ to $70 \mathrm{~Gy}$. In a meta-analysis comprising 14 studies, Burbach et al. [31] reported that doses of $\geq 60$ Gy were associated with an increased pCR rate with acceptable early toxicity. Notably, in the era of total mesorectal excision, dose escalation with the simple purpose of increasing the rate of pCR may not be beneficial because of the possible increase in toxicities without an increase in local control. Taking into consideration the importance of the circumferential resection margin status after PCRT for local recurrence [32], dose escalation with SIBIMRT would be helpful for patients with mesorectal fascia involvement at the time of diagnosis.

The rate of early leakage after sphincter-saving resection for rectal cancer has been reported to be 3\%-20\% [33,34]. In the present study, on using the current standard RT dose of 45-50 Gy, early leakage occurred in 8 patients (9\%), which was comparable to previous studies and was not different between the SIB-IMRT and 3D-CRT groups. However, there have been few reports about the relationship between postsurgical complications and escalated RT dose over 50 Gy in the setting of PCRT for LARC. Therefore, it is necessary to find the optimal RT dose for PCRT while considering both tumor response and toxicities.

There are several limitations of the current study. As a retrospective study, the small number of patients and uncontrolled characteristics related to tumors, patients, and treatments could have caused bias. For example, although all SIB-IMRT-group patients received $50 \mathrm{~Gy}$, only $50 \%$ of the 3D-CRT-group patients received $50 \mathrm{~Gy}$, which can make comparison of dose-volume parameters difficult. This difference could have also caused more $\mathrm{Gl}$ toxicities of higher grades in the SIB-IMRT group.

In the current study, SIB-IMRT achieved tumor response profiles comparable to those of $3 \mathrm{D}-\mathrm{CRT}$, using similar RT strategies. During RT, patients treated with SIB-IMRT experienced similar or reduced treatment-related toxicities compared with 3D-CRT treated patients. In addition, reduced fraction number with one simulation would be an advantage of SIB-IMRT. Long-term follow-up is needed to see the differences in the late toxicities.

\section{Conflict of Interest}

No potential conflict of interest relevant to this article was reported.

\section{Acknowledgements}

This research was supported by Kyungpook National University Research Fund, 2015.

\section{References}

1. Sauer R, Liersch T, Merkel S, et al. Preoperative versus postoperative chemoradiotherapy for locally advanced rectal cancer: results of the German CAO/ARO/AIO-94 randomized phase III trial after a median follow-up of 11 years. J Clin Oncol 2012;30:1926-33.

2. Gambacorta MA, Pasini D, Minsky BD, et al. Is two-dimensional field definition sufficient for pelvic node coverage in rectal cancer compared to technical three-dimensional definition? Tumori 2013;99:191-8.

3. Roels S, Duthoy W, Haustermans $K_{1}$ et al. Definition and delineation of the clinical target volume for rectal cancer. Int J Radiat Oncol Biol Phys 2006;65:1129-42.

4. Zhao J, Hu W, Cai G, et al. Dosimetric comparisons of VMAT, IMRT and 3DCRT for locally advanced rectal cancer with simultaneous integrated boost. Oncotarget 2016;7:6345-51.

5. $\mathrm{Yu} M$, Lee JH, Jang HS, et al. A comparison of dosimetric parameters between tomotherapy and three-dimensional conformal radiotherapy in rectal cancer. Radiat Oncol 2013;8:181.

6. Yu M, Jang HS, Jeon DM, et al. Dosimetric evaluation of Tomotherapy and four-box field conformal radiotherapy in locally advanced rectal cancer. Radiat Oncol J 2013;31:252-9.

7. Li XA, Wang JZ, Jursinic PA, Lawton CA, Wang D. Dosimetric 
advantages of IMRT simultaneous integrated boost for high-risk prostate cancer. Int J Radiat Oncol Biol Phys 2005;61:1251-7.

8. Dogan N, King S, Emami B, et al. Assessment of different IMRT boost delivery methods on target coverage and normal-tissue sparing. Int J Radiat Oncol Biol Phys 2003;57:1480-91.

9. Zhu J, Liu F, Gu W, et al. Concomitant boost IMRT-based neoadjuvant chemoradiotherapy for clinical stage II/III rectal adenocarcinoma: results of a phase II study. Radiat Oncol 2014;9:70.

10. Li JL, Ji JF, Cai $Y$, et al. Preoperative concomitant boost intensity-modulated radiotherapy with oral capecitabine in locally advanced mid-low rectal cancer: a phase II trial. Radiother Oncol 2012;102:4-9.

11. Hernando-Requejo O, Lopez M, Cubillo A, et al. Complete pathological responses in locally advanced rectal cancer after preoperative IMRT and integrated-boost chemoradiation. Strahlenther Onkol 2014;190:515-20.

12. Engels $B$, Platteaux N, Van den Begin $R$, et al. Preoperative intensity-modulated and image-guided radiotherapy with a simultaneous integrated boost in locally advanced rectal cancer: report on late toxicity and outcome. Radiother Oncol 2014;110:155-9.

13. Myerson RJ, Garofalo MC, El Naqa I, et al. Elective clinical target volumes for conformal therapy in anorectal cancer: a radiation therapy oncology group consensus panel contouring atlas. Int J Radiat Oncol Biol Phys 2009;74:824-30.

14. Dworak O, Keilholz L, Hoffmann A. Pathological features of rectal cancer after preoperative radiochemotherapy. Int J Colorectal Dis 1997;12:19-23.

15. Edge SB, Compton CC. The American Joint Committee on Cancer: the 7th edition of the AJCC cancer staging manual and the future of TNM. Ann Surg Oncol 2010;17:1471-4.

16. Janjan NA, Khoo VS, Abbruzzese J, et al. Tumor downstaging and sphincter preservation with preoperative chemoradiation in locally advanced rectal cancer: the M. D. Anderson Cancer Center experience. Int J Radiat Oncol Biol Phys 1999;44:102738.

17. de Campos-Lobato LF, Stocchi L, da Luz Moreira A, et al. Pathologic complete response after neoadjuvant treatment for rectal cancer decreases distant recurrence and could eradicate local recurrence. Ann Surg Oncol 2011;18:1590-8.

18. Kim TH, Chang HJ, Kim DY, et al. Pathologic nodal classification is the most discriminating prognostic factor for disease-free survival in rectal cancer patients treated with preoperative chemoradiotherapy and curative resection. Int J Radiat Oncol Biol Phys 2010;77:1158-65.
19. Rodel C, Martus P, Papadoupolos T, et al. Prognostic significance of tumor regression after preoperative chemoradiotherapy for rectal cancer. J Clin Oncol 2005;23:8688-96.

20. Park SH, Kim JC. Preoperative chemoradiation for locally advanced rectal cancer: comparison of three radiation dose and fractionation schedules. Radiat Oncol J 2016;34:96-105.

21. Huebner M, Wolff BG, Smyrk TC, Aakre J, Larson DW. Partial pathologic response and nodal status as most significant prognostic factors for advanced rectal cancer treated with preoperative chemoradiotherapy. World J Surg 2012;36:67583.

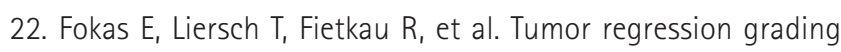
after preoperative chemoradiotherapy for locally advanced rectal carcinoma revisited: updated results of the CAO/ARO/ Al0-94 trial. J Clin Oncol 2014;32:1554-62.

23. Samuelian JM, Callister MD, Ashman JB, Young-Fadok TM, Borad MJ, Gunderson LL. Reduced acute bowel toxicity in patients treated with intensity-modulated radiotherapy for rectal cancer. Int J Radiat Oncol Biol Phys 2012;82:1981-7.

24. Parekh A, Truong MT, Pashtan I, et al. Acute gastrointestinal toxicity and tumor response with preoperative intensity modulated radiation therapy for rectal cancer. Gastrointest Cancer Res 2013;6:137-43.

25. Hong TS, Moughan J, Garofalo MC, et al. NRG Oncology Radiation Therapy Oncology Group 0822: a phase 2 study of preoperative chemoradiation therapy using intensity modulated radiation therapy in combination with capecitabine and oxaliplatin for patients with locally advanced rectal cancer. Int J Radiat Oncol Biol Phys 2015;93:29-36.

26. Baglan KL, Frazier RC, Yan D, Huang RR, Martinez AA, Robertson JM. The dose-volume relationship of acute small bowel toxicity from concurrent 5-FU-based chemotherapy and radiation therapy for rectal cancer. Int J Radiat Oncol Biol Phys 2002;52:176-83.

27. Viswanathan AN, Yorke ED, Marks LB, Eifel PJ, Shipley WU. Radiation dose-volume effects of the urinary bladder. Int $J$ Radiat Oncol Biol Phys 2010;76:S116-22.

28. Capirci C, Valentini V, Cionini L, et al. Prognostic value of pathologic complete response after neoadjuvant therapy in locally advanced rectal cancer: long-term analysis of 566 ypCR patients. Int J Radiat Oncol Biol Phys 2008;72:99-107.

29. Sanghera P, Wong DW, McConkey CC, Geh JI, Hartley A. Chemoradiotherapy for rectal cancer: an updated analysis of factors affecting pathological response. Clin Oncol (R Coll Radiol) 2008;20:176-83.

30. Appelt AL, Ploen J, Vogelius IR, Bentzen SM, Jakobsen A. Radiation dose-response model for locally advanced rectal 
cancer after preoperative chemoradiation therapy. Int J Radiat Oncol Biol Phys 2013;85:74-80.

31. Burbach JP, den Harder AM, Intven $M$, van Vulpen $M_{1}$ Verkooijen HM, Reerink 0 . Impact of radiotherapy boost on pathological complete response in patients with locally advanced rectal cancer: a systematic review and metaanalysis. Radiother Oncol 2014;113:1-9.

32. Nagtegaal ID, Quirke P. What is the role for the circumferential margin in the modern treatment of rectal cancer? J Clin Oncol
2008;26:303-12.

33. Yeh $C Y$, Changchien $C R$, Wang JY, et al. Pelvic drainage and other risk factors for leakage after elective anterior resection in rectal cancer patients: a prospective study of 978 patients. Ann Surg 2005;241:9-13.

34. Kim CW, Kim JH, Yu CS, et al. Complications after sphinctersaving resection in rectal cancer patients according to whether chemoradiotherapy is performed before or after surgery. Int J Radiat Oncol Biol Phys 2010;78:156-63. 